\title{
Detecting Transparent Barriers: Clear Evidence Against the Means-End Deficit Account of Search Failures
}

\author{
Jeanne L. Shinskey and Yuko Munakata \\ Department of Psychology \\ University of Denver
}

\begin{abstract}
The standard explanation of infants' search failures with hidden objects, despite an apparent sensitivity to them, is a deficit in the means-end skill for retrieving objects from occluders. Studies equating means-end demands for retrieving toys from transparent and opaque barriers challenge this account by showing that infants succeed more with visible objects. However, they suffer from a critical limitation: Infants may retrieve visible objects without noticing the transparent barriers in front of them. We addressed this concern by requiring infants to notice a barrier to retrieve a toy and specifically to pull down a rotating screen to retrieve a toy from behind it. Seven-month-olds used this means-end skill more often with a transparent barrier than an opaque one. Thus, neither a means-end deficit nor an ability to ignore transparent barriers fully accounts for search failures. Relations to other findings challenging the means-end deficit account and implications for approaches to studying cognitive development are discussed.
\end{abstract}

Why do infants fail to search for hidden objects before 8 to 10 months when they seem sensitive to hidden objects months earlier in other measures? The prevailing explanation is that infants lack the means-end skill for retrieving objects from occluders. Recent evidence challenges this means-end deficit account but suffers from a specific limitation. The study presented here addresses this limitation, definitively challenging the means-end deficit explanation and highlighting the need for theoretical accounts that address both successes and failures with hidden objects.

The traditional explanation for infants' search failures before 8 to 10 months was that they lack the concept of object permanence (Piaget, 1954). However,

Requests for reprints should be sent to Jeanne L. Shinskey or Yuko Munakata, Department of Psychology, University of Denver, 2155 South Race Street, Denver, CO 80208-2478. 
manual search may be inadequate for measuring young infants' knowledge; search may be too difficult because of other limited abilities. Because of this criticism, researchers have used other paradigms to investigate infants' knowledge about hidden objects. In violation-of-expectation studies, infants watch events in which hidden objects undergo either possible or impossible transformations while their looking times to the events are measured (e.g., Baillargeon, 1993; Spelke, Breinlinger, Macomber, \& Jacobson, 1992; Wynn, 1992). In many such studies, infants as young as 3.5 months look longer at impossible than possible events. The conclusion is that infants know hidden objects exist, are surprised to see their existence violated, and therefore look longer. Thus, when reaching is not required, infants seem sensitive to hidden objects.

Why, then, do infants fail to search for hidden objects until months later? According to the prevailing means-end deficit account, infants know the object continues to exist but cannot coordinate the actions to uncover it in a means-end sequence (e.g., Baillargeon, Graber, DeVos, \& Black, 1990; Bower \& Wishart, 1972; Diamond, 1991). The hidden object is the end, and removing the occluder is the means. Infants' reaching in the dark supports the means-end deficit account. Infants reach for objects concealed by darkness, which allows a direct reach rather than requiring a means-end sequence, before they reach for objects occluded by a barrier in the light (e.g., Clifton, Rochat, Litovsky, \& Perris, 1991; Hood \& Willatts, 1986).

Despite this supportive evidence, however, other evidence suggests a means-end deficit cannot be the sole cause of infants' failures in some tasks and successes in others. Even when means-end demands are equated for retrieving visible and hidden toys, infants retrieve visible toys more often and demonstrate little sensitivity to hidden toys. One approach equates means-end demands by introducing them into a visible condition. If infants demonstrate means-end skill with a transparent barrier, then failure with an opaque barrier cannot be due solely to a means-end deficit. Infants who watch as objects are placed behind transparent and opaque barriers retrieve them more often in the transparent condition (e.g., Bower \& Wishart, 1972; Munakata, McClelland, Johnson, \& Siegler, 1997; Shinskey, Bogartz, \& Poirier, 2000). Another approach equates means-end demands by taking them away from both visible and hidden events. For example, 6-month-olds presented with objects visible in water or hidden in milk retrieved visible objects more often, even though they could reach directly in both cases (Shinskey, 2000). This collection of results challenges the means-end deficit account. Such patterns are not observed when the behavior does not serve as a means to an end (Munakata, Bauer, Stackhouse, \& Tobiasson, 2000), indicating that the behaviors are truly means-end in nature rather than reflecting simple arousal or dishabituation.

However, previous studies equating means-end demands for visible and hidden events suffer from a critical limitation: The means-end demands may not be truly equated. Infants could succeed at retrieval without even noticing the transparent barrier. For example, an infant presented with an object under a transparent cup may try 
to reach directly for the object without noticing the cup, inadvertently displace the cup, and then retrieve the toy (Munakata et al., 1997). This is unlikely to occur with an opaque cup, which cannot be ignored like a transparent one. Although Munakata et al. (1997) controlled for such inadvertent retrievals by having the toy beyond reach, the visible event may appear simpler because the transparent screen can be ignored, giving infants one less thing to think about during visible events (E. Spelke, personal communication, April 1995). Thus, in all previous studies in which means-end demands for retrieving visible and occluded objects were ostensibly equated, the demands may have been higher in the occluded case; as a result, means-end limitations may have prevented infants from retrieving hidden objects.

We addressed this concern by creating a transparent event in which infants had to notice and use a drawbridge-like screen as a means to get a toy. If they ignored the screen, they failed to get the toy. Seven-month-old infants witnessed events in which either a toy or no toy was placed behind either a transparent or an opaque screen, and we measured how often they pulled down the screen. According to the means-end deficit account, infants should discriminate similarly between toy presence and absence with the transparent and opaque screens because the two screens require the same means-end skill. In contrast, we predicted that infants would discriminate more between toy presence and absence when the screen was transparent than when it was opaque.

\section{METHOD}

\section{Participants}

Sixteen full-term 7-month-old infants ( $M=7$ months, 3 days; range $=6 ; 27$ to $7 ; 8 ; 8$ girls and 8 boys) from the Denver, Colorado, area participated. Sixteen additional infants were tested but not included in the sample because of fussiness (5), failure to pull the screen down during demonstration (4), or failure to pull the screen down within $5 \mathrm{sec}$ on three trials during familiarization (7). ${ }^{1}$

\section{Apparatus, Stimuli, and Equipment}

The two apparati were identical except the screen was transparent in one and opaque in the other (see Figure 1). The base of each was a piece of gray-painted ply-

\footnotetext{
${ }^{1}$ The high rate of attrition during the demonstration and training phases might seem to support the means-end deficit account. However, there was no means-end problem to solve during training because the toy had not yet been introduced. Moreover, the attrition rate does not weaken the argument that even after infants passed the demonstration and training criteria, other factors prevented them from searching for hidden toys.
} 

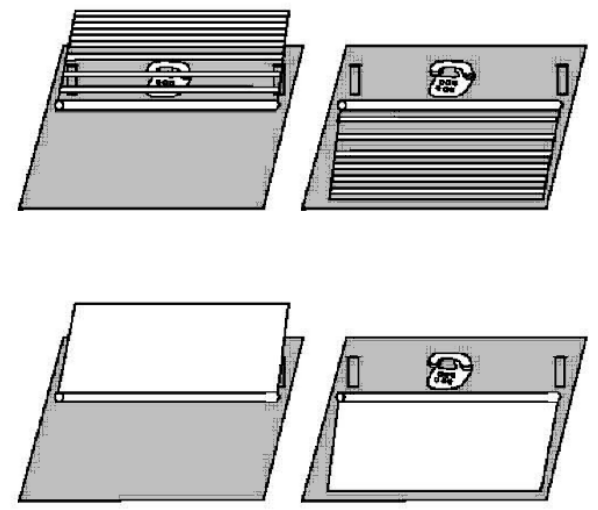

FIGURE 1 The transparent and opaque apparati with the screens in both up and down positions.

wood $(33 \mathrm{~cm} \times 13 \mathrm{~cm})$. The reverse side of the base was covered with gray felt. Two black-painted wooden tracks $(1.5 \mathrm{~cm} \times 45 \mathrm{~cm} \times 0.75 \mathrm{~cm})$ constrained the motion of the apparatus. Each screen was glued into a $25.5-\mathrm{cm}$ groove cut into a gray-painted dowel $(29.5 \mathrm{~cm} \times 1 \mathrm{~cm})$ placed across the center of the base. The ends of the dowel rested in 1-cm holes cut into two gray-painted wooden blocks $(2 \mathrm{~cm} \times 7.5 \mathrm{~cm} \times 6.5$ $\mathrm{cm}$ ) nailed to the left and right sides of the base. The dowel rested approximately 0.5 $\mathrm{cm}$ above the base.

The motion of the screen from $0^{\circ}$ to $115^{\circ}$ was stopped by a $0.5 \mathrm{~cm} \times 3.75 \mathrm{~cm}$ piece of clear plastic tubing glued to the left block. The transparent screen $(25.5 \mathrm{~cm}$ $\times 12.5 \mathrm{~cm}$ ) was a piece of Plexiglas covered with clear laminating plastic and ten $0.5 \mathrm{~cm} \times 25.5 \mathrm{~cm}$ horizontal stripes of white contact paper $0.5 \mathrm{~cm}$ apart. The opaque screen $(25.5 \mathrm{~cm} \times 12.5 \mathrm{~cm})$ consisted of Plexiglas covered by white contact paper. A gray-painted wooden toy platform $(6.5 \mathrm{~cm} \times 7.5 \mathrm{~cm} \times 2 \mathrm{~cm}), 5 \mathrm{~cm}$ behind the dowel and centered in the base, prevented the dowel from obstructing the infant's view of the toy. The stimuli were 10 toys approximately $6 \mathrm{~cm} \times 5 \mathrm{~cm} \times 6 \mathrm{~cm}$.

The apparati were specifically designed to prevent infants from retrieving the toy with a direct reach. The $115^{\circ}$ angle of the screens required infants to pull the screen toward them. The screens were large enough so that infants who reached directly for the visible toy in the center would not accidentally contact the top or side edges of the screen, which were the means to pulling the screen down.

The experimenter timed the events using an audiotape of a metronome that beeped once per second. A videocamera recorded the infant.

\section{Design and Procedure}

We used a 2 (familiarization condition) $\times 2$ (testing condition $) \times 2$ (screen type $) \times 2$ (toy presence) factorial design, with familiarization and testing conditions as be- 
tween-participant factors and screen type and toy presence as within-participants factors. The two familiarization orders counterbalanced the screen with which the infant was familiarized first. The two testing orders counterbalanced toys with screen type: All participants saw each toy on the same trial, but half saw it with the transparent screen and half with the opaque screen. Infants had five repeated trials of the four test events in blocks of four, with random event order within each block, for a total of 20 test trials.

The infant sat in an infant seat fastened to the table, with the parent directly behind. We familiarized infants with each screen in four phases prior to test. A demonstration phase introduced the apparatus and allowed the infant to manipulate it. A training phase familiarized infants with how to pull the screen down. A habituation phase bored infants with pulling the screen down to reduce the possibility that infants would pull the screen down on test trials merely because they were still interested in playing with the screen rather than as a means to get the toy. Finally, a toy phase familiarized infants with the resting location of the toy on test trials. Infants first received the demonstration and training phases with one screen, followed by the other screen. Infants then received the habituation and toy phases with the first screen, followed by the second.

The demonstration phase began with the apparatus in the center of the table and the screen raised. The experimenter moved the screen down and up until the infant attended to it for several passes. The experimenter left the screen raised and pushed the apparatus to the infant $(2.5 \mathrm{sec})$. If the infant hesitated to explore the apparatus, the experimenter asked the parent to encourage the infant to pull the screen down. There was no time limit. The demonstration phase ended when the infant pulled the screen down past the point where he or she could see the toy platform over the top of the screen.

In the training phase, each trial began with the apparatus in the center of the table and the screen lying flat. The experimenter tapped her fingers behind the toy platform until the infant looked, while calling the infant by name. The experimenter then raised the screen and pushed the apparatus to the infant. When the apparatus stopped moving, the experimenter began to time the trial. Each trial ended when the infant pulled the screen down or $5 \mathrm{sec}$ elapsed. If the infant failed to pull the screen down after several trials, the experimenter let the trial continue longer than $5 \mathrm{sec}$, repeated the demonstration phase, or asked the parent to encourage the infant to pull the screen down. The training phase ended after the infant pulled the screen down within $5 \mathrm{sec}$ on any three trials without assistance.

Habituation trials were identical to training trials. Habituation ended when the infant failed to pull the screen down for two consecutive trials, or after 12 trials passed.

The toy phase began with the apparatus at the center of the table and the screen lying flat. The experimenter placed a toy on the toy platform and tapped her fingers 
behind the toy platform until the infant looked, while calling the infant by name. The apparatus was pushed to the infant, with the screen remaining flat, allowing the infant to reach directly for the toy. There was no time limit.

There were four test events: transparent-toy, transparent-no toy, opaque-toy and opaque-no toy. Toy trials began with the placement of the toy on the toy platform and were otherwise identical to training and habituation trials. No-toy trials were identical to training and habituation trials. Toy trials ended when $5 \mathrm{sec}$ elapsed or when the infant pulled the screen down. No-toy trials ended when $5 \mathrm{sec}$ elapsed regardless of whether the infant pulled the screen down, to avoid conditioning infants to reach at ceiling levels by rewarding them with the end of the trial as soon as they pulled the screen down.

\section{Measures and Interobserver Reliability}

The main dependent measure assessed whether or not the infant pulled the screen down far enough to see over the top to the toy platform. Retrieval was not used as the dependent measure because there was no toy on half the trials. To address any concerns that infants who reached directly for the toy behind the transparent screen might reflexively grasp the screen and unintentionally pull it down, direct reaches were also measured on transparent-toy trials. A direct reach consisted of extending one or both hands toward the screen in the center, near the object, rather than near the top or side edges. For both measures, two observers coded all trials for half the infants. Agreement was $99 \%$ for pulling the screen down (i.e., 158 of 160 judgments) and $93 \%$ for direct reaches (i.e., 37 of 40 judgments).

\section{RESULTS}

A 2 (familiarization condition) $\times 2$ (testing condition) $\times 2$ (screen type) $\times 2$ (toy presence) analysis of variance (ANOVA) on the dependent measure of pulling the screen down revealed an interaction of screen type and toy presence, $F(1,12)=$ $6.33, p<.05$. As predicted, infants discriminated more between toy presence and toy absence with the transparent than the opaque screen (left side of Figure 2). Infants pulled the screen down on $82 \%(S E=6.80)$ of transparent-toy trials and $44 \%$ $(S E=7.12)$ of transparent-no toy trials. In contrast, the values were $51 \%(S E=$ $8.56)$ for opaque-toy trials and $39 \%(S E=7.18)$ for opaque-no toy trials. A main effect of toy presence also demonstrated that infants pulled the screens down more often when a toy was present $(M=67 \%, S E=6.50)$ than when it was absent $(M=$ $41 \%, S E=6.45), F(1,12)=15.14, p<.01$. A main effect of screen type also indicated that infants pulled down the transparent screen $(M=63 \%, S E=5.53)$ more 


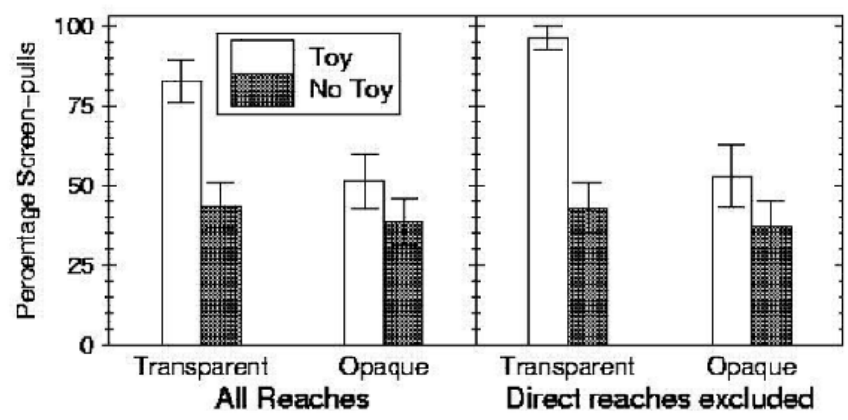

FIGURE 2 Percentage of trials on which infants pulled the screen down, by screen type and toy presence (left side of figure). The same pattern emerged when direct reaches were excluded (right side of figure).

than the opaque screen $(M=45 \%, S E=6.39), F(1,12)=11.63, p<.01 .{ }^{2}$ No other main effects or interactions were significant (all $F \mathrm{~s}<2$ ).

To address any concerns that infants may have inadvertently pulled the transparent screen down while attempting to reach directly through the screen for the object, trials on which such direct reaches occurred were excluded in a subsequent ANOVA. The same pattern of results emerged (right side of Figure 2). There was a significant interaction of screen type and toy presence, $F(1,10)=$ $7.87, p<.05$. Infants pulled the screen down on 96\% ( $S E=3.57)$ of transparent-toy trials and $43 \%(S E=8.08)$ of transparent-no toy trials. In contrast, the values were $53 \%(S E=9.75)$ for opaque-toy trials and $37 \%(S E=8.08)$ for opaque-no toy trials. A main effect of toy presence indicated that infants pulled the screens down more often when a toy was present $(M=75 \%, S E=5.85)$ than when it was absent $(M=40 \%, S E=7.34), F(1,10)=32.25, p<.001$. A main effect of screen type also revealed that infants pulled down the transparent screen $(M=70 \%, S E=4.76)$ more than the opaque screen $(M=45 \%, S E=7.90), F(1$, $10)=22.81, p<.01$. No other main effects or interactions were significant (all $F \mathrm{~s}<2$ ). There was no evidence to indicate that infants received an unfair advantage in the transparent case by pulling the screen down unintentionally while attempting to reach directly for the toy.

\footnotetext{
${ }^{2}$ Infants did not simply find the transparent screen inherently more interesting. Analyses of training and habituation data revealed that infants took no longer to reach the training criterion or the habituation criterion with one screen than with the other (all $F \mathrm{~s}<3$ ). The main effect of screen during test trials results from the interaction: Infants pulled the screen down more in the transparent-toy condition than in the other three conditions.
} 


\section{DISCUSSION}

The major finding was that even when infants could not ignore the transparent screen, they discriminated more between toy presence and toy absence with the transparent screen than with the opaque one. Thus, failure to retrieve hidden objects cannot be due solely to a means-end deficit or to an ability to ignore transparent barriers. These findings are not unique to this particular means-end task. Infants retrieve objects more often from transparent barriers than from opaque barriers regardless of whether they remove a cover, pull a towel, push a button, reach through a curtain, or reach into liquids (Bower \& Wishart, 1972; Munakata et al., 1997; Shinskey, 2000). Furthermore, the experiment controlled for factors that previous research with transparent barriers has not. Specifically, infants could not ignore the transparent barrier and still succeed in retrieving the object. These results address the limitations of previous studies to challenge the means-end deficit account.

We stress that our results demonstrate that means-end deficits cannot be the sole source of infants' search failures, not that such deficits play no role in search failures. Such deficits may play a more critical role for infants younger than 7 months. Nevertheless, even after infants master the required means-end skills, they use them less with hidden than visible objects. Something other than means-end deficits must contribute to infants' search failures. Thus, despite the prevalence of the means-end account of infants' search failures, the puzzle remains regarding why infants simultaneously succeed and fail at different tasks designed to measure the same knowledge of hidden objects.

Theories of cognitive development must be broad enough to reconcile such simultaneous successes and failures. Our results challenge approaches that attribute early sensitivity to innate knowledge or highly constrained learning mechanisms and discount failures as due to ancillary deficits (Baillargeon, 1994; Diamond, 1991). Several alternative approaches exist. According to the graded representations account (e.g., Munakata et al., 1997), infants' mental representations of hidden objects strengthen with development; infants can demonstrate sensitivity to hidden objects in violation-of-expectation studies with a relatively weak representation but need a stronger representation to guide action in a reaching task. Likewise, infants may require stronger representations to search for objects behind occluders than to search for them in the dark because occluders provide visible evidence contradicting the presence of the hidden object, whereas there is simply no visible evidence in the dark. In other words, the memory demands on the representations may be greater in the light than in the dark. Alternatively, different neural pathways may account for the differences in looking and reaching: An early-developing visual pathway may guide looking, whereas a later-developing action pathway may guide reaching (e.g., Bertenthal, 1996; Spelke, Vishton, \& von Hofsten, 1995). A related possibility is that looking and reaching tasks draw on two distinct types of representations, rather than one ranging along a continuum (e.g., Munakata, 2001; Schacter \& Moscovitch, 
1984). Further research exploring these approaches may advance our understanding of the nature of early cognitive development.

\section{ACKNOWLEDGMENTS}

The study was supported by grants from the National Institute for Child Health and Development (1R29 HD37163-01) and the National Science Foundation (IBN-9873492). We thank Elizabeth Weinberger for help in apparatus construction and scheduling appointments with parents, Jeremy Weltman for help in apparatus construction, Mario Garcia for interobserver reliability coding, and the members of the Cognitive Development Center for their helpful feedback.

\section{REFERENCES}

Baillargeon, R. (1993). The object concept revisited: New directions in the investigation of infants' physical knowledge. In C. Granrud (Ed.), Visual perception and cognition in infancy (pp. 265-315). Hillsdale, NJ: Lawrence Erlbaum Associates, Inc.

Baillargeon, R. (1994). How do infants learn about the physical world? Current Directions in Psychological Science, 3, 133-139.

Baillargeon, R., Graber, M., DeVos, J., \& Black, J. (1990). Why do young infants fail to search for hidden objects? Cognition, 36, 255-284.

Bertenthal, B. I. (1996). Origins and early development of perception, action, and representation. Annual Review of Psychology, 47, 431-459.

Bower, T. G. R., \& Wishart, J. G. (1972). The effects of motor skill on object permanence. Cognition, 1, $165-172$.

Clifton, R. K., Rochat, P., Litovsky, R. Y., \& Perris, E. E. (1991). Object representation guides infants' reaching in the dark. Journal of Experimental Psychology, 17, 323-329.

Diamond, A. (1991). Neuropsychological insights into the meaning of object concept development. In S. Carey \& R. Gelman (Eds.), The epigenesis of mind (pp. 67-110). Hillsdale, NJ: Lawrence Erlbaum Associates, Inc.

Hood, B., \& Willatts, P. (1986). Reaching in the dark to an object's remembered position: Evidence for object permanence in 5-month-old infants. British Journal of Developmental Psychology, 4, 57-65.

Munakata, Y. (2001). Task-dependency in infant behavior: Toward an understanding of the processes underlying cognitive development. In F. Lacerda, C. von Hofsten, \& M. Heimann (Eds.), Emerging cognitive abilities in early infancy (pp. 29-52). Mahwah, NJ: Lawrence Erlbaum Associates, Inc.

Munakata, Y., Bauer, D., Stackhouse, T., \& Tobiasson, J. (2000). Rich interpretation vs. deflationary accounts in cognitive development: The case of means-ends skills in 7-month-old infants. Manuscript submitted for publication.

Munakata, Y., McClelland, J. L., Johnson, M. H., \& Siegler, R. S. (1997). Rethinking infant knowledge: Toward an adaptive process account of successes and failures in object permanence tasks. Psychological Review, 104, 686-713.

Piaget, J. (1954). The construction of reality in the child. New York: Basic.

Schacter, D., \& Moscovitch, M. (1984). Infants, amnesics, and dissociable memory systems. In M. Moscovitch (Ed.), Advances in the study of communication and affect: Vol. 9. Infant memory (pp. 173-216). New York: Plenum. 


\section{SHINSKEY AND MUNAKATA}

Shinskey, J. L. (2000). Infants' search failures: Can we still milk the means-end deficit account? Manuscript submitted for publication.

Shinskey, J. L., Bogartz, R. S., \& Poirier, C. R. (2000). The effects of graded occlusion on manual search and visual attention in 5- to 8-month-old infants. Infancy, 1, 323-346.

Spelke, E., Breinlinger, K., Macomber, J., \& Jacobson, K. (1992). Origins of knowledge. Psychological Review, 99, 605-632.

Spelke, E. S., Vishton, P., \& von Hofsten, C. (1995). Object perception, object-directed action, and physical knowledge in infancy. In M. Gazzaniga (Ed.), The cognitive neurosciences (pp. 165-179). Cambridge, MA: MIT Press.

Wynn, K. (1992). Addition and subtraction by human infants. Nature, 358, 749-750. 